\title{
UPSCALING OF SOLAR INDUCED CHLOROPHYLL FLUORESCENCE FROM LEAF TO CANOPY USING THE DART MODEL AND A REALISTIC 3D FOREST SCENE
}

\author{
Weiwei Liu ${ }^{\text {abc }}$, Jon Atherton ${ }^{\mathrm{c}}$, Matti Mõttus ${ }^{\mathrm{d}}$, Alasdair MacArthur ${ }^{\mathrm{e}}$, Hakala Teemu ${ }^{\mathrm{f}}$, Kadmiel Maseyk ${ }^{\mathrm{g}}$, Iain Robinson ${ }^{\mathrm{h}}$, Eija \\ Honkavaara $^{\mathrm{f}}$, Albert Porcar-Castell ${ }^{\mathrm{c}, *}$ \\ ${ }^{a}$ LREIS, Institute of Geographic Sciences and Natural Resources Research, Chinese Academy of Sciences, 100101, China - \\ lww@1reis.ac.cn \\ b University of Chinese Academy of Sciences, Beijing 100049, China - lww@lreis.ac.cn \\ c Optics of Photosynthesis Laboratory, Department of Forest Sciences, University of Helsinki, PO Box 27, 00014 Helsinki, Finland \\ - (weiwei.liu, jon.atherton, joan.porcar)@helsinki.fi \\ d VTT Techical Research Centre of Finland, PO Box 1000, 02044 VTT, Finland - matti.mottus@ gmail.com \\ ${ }^{\text {e }}$ School of Geosciences, University of Edinburgh, Grant Institute, Edinburgh. EH9 3FF, U.K - alasdair.macarthur@ed.ac.uk \\ ${ }^{\mathrm{f}}$ Finnish Geodetic Institute, Geodeetinrinne 2, P.O. Box 15, 02431 Masala, Finland - (teemu.hakala, eija.honkavaara)@nls.fi \\ g Department of Environment, Earth and Ecosystems, Faculty of Science, Open University, Milton Keynes, U.K - \\ kadmiel.maseyk@open.ac.uk \\ ${ }^{\text {h }}$ Laser Spectroscopy Group, Rutherford Appleton Laboratory, U.K - iain@physics.org
}

\section{Commission III, WG III/4}

KEY WORDS: Chlorophyll florescence, Upscaling, DART, 3D scene, Forest

\begin{abstract}
:
Solar induced chlorophyll a fluorescence (SIF) has been shown to be an excellent proxy of photosynthesis at multiple scales. However, the mechanical linkages between fluorescence and photosynthesis at the leaf level cannot be directly applied at canopy or field scales, as the larger scale SIF emission depends on canopy structure. This is especially true for the forest canopies characterized by high horizontal and vertical heterogeneity. While most of the current studies on SIF radiative transfer in plant canopies are based on the assumption of a homogeneous canopy, recently codes have been developed capable of simulation of fluorescence signal in explicit 3-D forest canopies. Here we present a canopy SIF upscaling method consisting of the integration of the 3-D radiative transfer model DART and a 3-D object model BLENDER. Our aim was to better understand the effect of boreal forest canopy structure on SIF for a spatially explicit forest canopy.
\end{abstract}

\section{INTRODUCTION}

Solar induced chlorophyll fluorescence (SIF) is an electromagnetic signal emitted by plants in the red and nearinfrared wavelengths by plants. Although measureable from space and aircraft, SIF originates close to the site of the photosynthetic reactions, and is therefore closely linked to plant growth and physiological status. Unlike the traditional reflectance vegetation indices, such as the Normalized Different Vegetation Index (NDVI), SIF responds very rapidly to environmental stress, and therefore has the potential to track time-varying stress at (sub) diurnal scales (Amoros-Lopez et al., 2008; Yang et al., 2015; Alonso et al., 2017).

The magnitude of SIF is very small compared to the radiation reflected by forest canopies, only $1-5 \%$ of reflected sunlight in the near infrared (Meroni et al., 2008). Additionally under natural illumination SIF cannot be measured directly due to the spectral overlap with reflectance, hence SIF is estimated using the Fraunhofer Line Depth (FLD) technique within narrow spectral windows, called Fraunhofer or telluric absorption bands (Alonso et al., 2008). At the leaf scale, spectral fluorescence is not only influenced by the dynamics of photosynthesis but also by chlorophyll content and leaf structure (Van Wittenberghe et al., 2015), while at the canopy scale, SIF estimated using the FLD technique is influenced by 1) sun and view geometry, 2) leaf and canopy structure and 3) background information (Damm et al., 2015).

In recent years, SIF has been used to calculate gross primary production (GPP), a key variable in carbon cycle and climate change studies. However, the relationship between SIF and GPP in these studies is empirical and likely to be at least partially dependent on the land surface and/or dominant vegetation types. According to previous research approximately $80 \%$ variability of canopy SIF is attributed to the leaf optical properties and canopy structure (Verrelst et al., 2015). Hence more research is required to disentangle the complex relationship between SIF and photosynthesis (such as the GPP), and frameworks coupling leaf fluorescence models and leafcanopy radiative transfer are required for better interpretation of plant biophysical characterization from SIF observations.

\footnotetext{
* Corresponding author
} 
Early attempts at using physically based models to simulate SIF were carried by the European Space Agency (ESA), in this work a leaf biophysical model FluorMODleaf and leaf-canopy fluorescence model FluorSAIL was developed (Meroni et al., 2004; Verhoef et al., 2004). Additionally, a more comprehensive model system, SCOPE (Soil-Canopy Observation, Photosynthesis and Energy Balance) was developed as fundamental research of the Fluorescence Explorer mission (FLEX). In SCOPE, SIF is coupled with the Soil-Vegetation-Atmosphere (SVAT) model capable of simulating photosynthesis, thermal radiation and energy balance (Van der Tol et al., 2009). However, all these works were based on the turbid medium SAIL model, which is a onedimensional RT model and not wholly suitable for applications to complex vegetation canopies, especially for forest canopies characterized by high horizontal and vertical heterogeneity (Porcar-Castell et al., 2014; Migliavacca et al., 2017).

Recently, 3-D radiative transfer model codes have been developed to address the need for realistic SIF simulations from complex canopy architectures. FluorWPS is a 3-D radiative transfer model based on the Monte Carlo ray-tracing method, and has the ability to model canopy SIF emitted from the complex canopies, such as the row-planted canopies (soybean and cotton) (Feng Zhao et al., 2016). Another model, the FluorFLIGHT 3-D radiative transfer model was also developed under this condition, which couples the 3-D raytracing forest radiative transfer model FLIGHT and leaf fluorescence model FLUSPECT. The FLIGHT model is a widely used 3-D vegetation reflectance model and can be used to simulate multiple scattering within the crown and the gap between the crown and other medium (Hernández-Clemente $\mathrm{R}$ et al., 2017; North, 1996). The results of FluorFLIGHT showed that the canopy SIF signal is greatly influenced for these complex canopy types with heterogeneity both in horizontal and vertical dimensions. However, as far as we are aware there are not yet any comprehensive studies that use 3-D modelling to test the response of SIF to canopy complexity in boreal type forests, such as those found in Finland.

The objective of this research was to evaluate a canopy SIF upscaling method considering the effect of complex forest structure, including leaf clumping, for a realistic forest scene.

\section{METHODOLOGY}

\subsection{Field data acquisition}

Measurements were conducted in a circular plot $(20 \mathrm{~m}$ in diameter) dominated by Silver birch (Betula pendula Roth), this stand is located in the Station for Measuring EcosystemAtmosphere Relations II (SMEAR II), Finland. Birch was selected for this study because it has significant heterogeneity in both horizontal and vertical directions. Survey data of every tree including the coordinates, tree height, crown height and DBH (Diameter at Breast Height) were collected in the field. Ground and bark reflectance data were measured in situ by a FileldSpec-HH spectrometer (ASD, Panalytical Devices, Ltd) with a sampling interval of $1.6 \mathrm{~nm}$ and spectral resolution of $3.5 \mathrm{~nm}$. Measurements of leaf reflectance and transmission was carried out in lab using an integrating sphere (Adapa Sphere, LabSphere, North Sutton, NH, USA) coupled with the FileldSpec-HH spectrometer.

\subsection{Upscaling approach}

In an attempt to simulate the SIF radiative transfer in heterogeneous canopies, we used the Discrete Anisotropic Radiative Transfer (DART), a comprehensive physically based 3-D RT model, which is designed to simulate radiation propagation from visible to thermal infrared in complex and heterogeneous 3-D landscape scenes, such as forest scenes and urban landscapes with topography and atmosphere (GastelluEtchegorry et al., 2017). The 3-D scene in DART is composed of an array of cells (i.e., voxels), and cells can be identified as either turbid matter or planar elements. Turbid matter is used to simulate homogeneous scenes, such as water, air and homogeneous vegetation, while the planar elements are used to simulate house, forest and other geometric objects. As one of the most comprehensive models for 3-D radiative transfer, DART has been used to simulate forest canopies (Malenovsky et al., 2008), and the simulation accuracy of canopy directional reflectance was tested through a comparison with other radiative transfer model based on in situ data (Widlowski $\mathrm{J}$ L et al., 2015).

The Fluspect RT model (Van der Tol et al., 2011) simulates leaf backward and forward chlorophyll a fluorescence from 400 to $850 \mathrm{~nm}$ of photosystems PSI and PSII, which are typically mixed together as a single emission spectrum in both leaf level spectral and canopy scale SIF measurements. Fluspect was implemented in DART's latest version, and was developed based on the widely used PROSPECT model and uses the socalled plate theory (Jacquemoud et al., 2009). As a first approximation the biochemical parameters and the fluorescence quantum efficiency was set to their default value.

The integration of DART and Fluspect model provides a solution for the demands of SIF upscaling in complex vegetation canopy (such as the boreal forest). But there were a few limitations. For example due to the constraints in the current version of DART, the whole scene had to consist of individual facets (Polygon) as turbid vegetation (Random distribution of infinitely small planar elements) cannot be currently used in SIF simulations.

\subsection{3-D simulation scene}

A simulation scene was created using two steps in this study. In the first step, a single tree (Figure 1) was created by the Blender software, which is a free and open source 3-D creation software, it supports 3-D object modelling with a set of groups of triangles (Allan Brito, 2008). A 3-D tree was created by the sapling tree gen module of blender with a height of $17.5 \mathrm{~m}$ and diameter of crown of $2.8 \mathrm{~m}$, which were average values from the survey data. An ellipsoid was selected to describe the crown shape, and a spherical distribution was assumed for the leaf inclination angle. In the second step, the trees created in Blender were saved as an OBJ format file, which is compatible with DART model, then a 3-D forest scene (Figure 2) was created in the DART model with the single tree modelled by Blender, using actual tree coordinates data from our field survey data.

In order to investigate the background effect on SIF canopy modeling, a 3-D grass layer (10 centimeter in thickness, $\mathrm{LAI}=1$ ) was created to simulate the background SIF emitted from the grass under the tree, the grass layer was composed of a set of 
groups of triangles that represent the leaves of grass. These triangles were randomly distributed in space. A spherical leaf angle distribution was used. The grass leaves were given the same optical and fluorescence parameters as the tree leaves. The simulation are for the nadir viewing geometry, with a fixed solar zenith angle of $30^{\circ}$ and solar azimuth angle of $223^{\circ}$. Additionally, the atmospheric impact has not been investigated in this study.

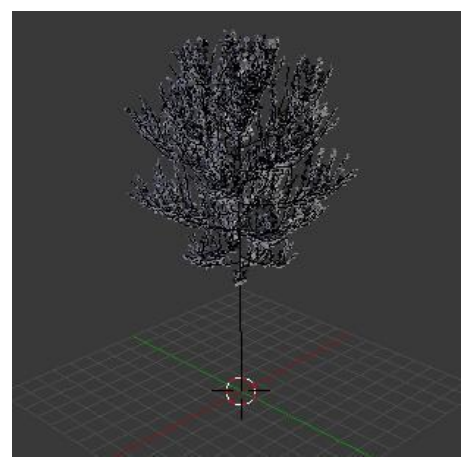

Figure 1. 3-D tree created by the Blender

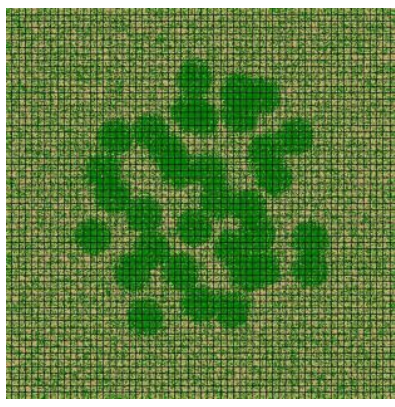

(a)

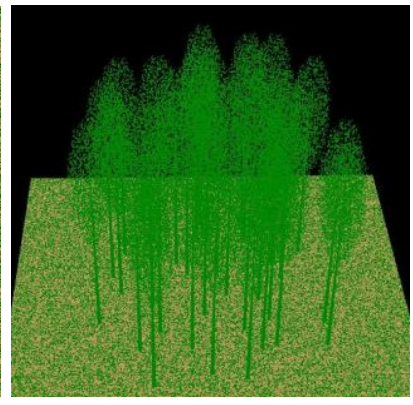

(b)
Figure 2. Forest 3-D scene: (a) Nadir view. (b) Oblique view

\section{RESULTS}

The number of elements in the forest scene used in this paper was about 7 million. It took about five hours to run the DART model in a ThinkPad laptop equipped with Intel i74510U Processor and 8GB RAM.

The SIF image at $740 \mathrm{~nm}$ was simulated by DART model with $0.5 \mathrm{~m}$ spatial resolution and $1 \mathrm{~nm}$ spectral resolution, a higher spatial and spectral resolution image can also be simulated according to demand. A SIF image of the sub scene (a square with $10 \mathrm{~m}$ in length) is shown in figure 4 . Figure 4 (a) shows the crown mask map in vertical direction, white pixels mean grass background, and the green pixels mean grass background covered with tree crown in vertical direction. A SIF image at $740 \mathrm{~nm}$ is shown in figure 4 (b). The results show that SIF value of crown-covered area is higher than the grass-covered area evidently with the hypothesis that grass and tree leaves have same optical and fluorescence properties.

We also simulated the full-spectrum canopy-reflected radiance and SIF, which is useful to better understand the SIF information from full emission spectrum, and also facilitates the integration of SIF with other optical reflectance index (such as the NDVI) and photochemical reflectance index (PRI). Figure 5 gives the result of full-spectrum simulation at canopy scale, the proposed method, which separates canopy and background vegetative layers, facilitates also the quantitative study of the impact of ground vegetation to total canopy SIF signal.

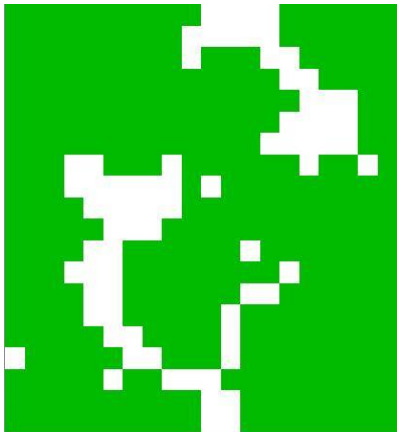

(a)

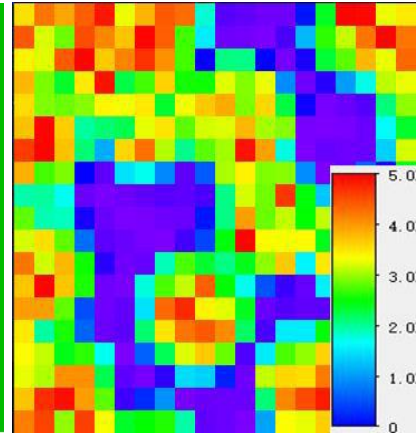

(b)
Figure 4. (a) Tree crown mask. (b) SIF image at 740nm (Unit: $\left.W / m^{2} / \mu m / s r\right)$

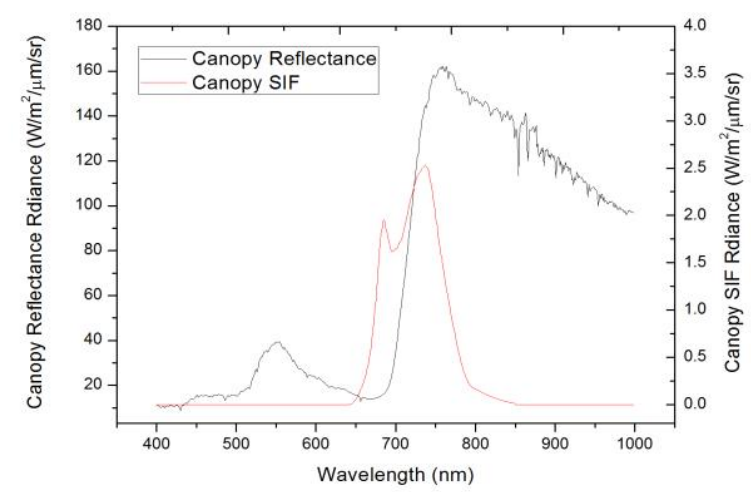

Figure 5. Canopy reflectance and SIF spectrum

\section{DISCUSSION AND CONCLUSION}

In this paper, a SIF upscaling method which combined DART, Fluspect and Blender was used for simulating 3-D heterogeneous forest canopies. Through the example of a SIF simulation in a birch forest, this approach has demonstrated the potential for simulating canopy SIF in semi-realistic boreal forest scenes.

The traditional 3-D tree used for radiative transfer modeling is a polygon, with leaves distributed at random within the polygon. Compared to the traditional 3-D radiative transfer modeling method, more detailed descriptions of spatial characteristics of the trees were created here using Blender, which enabled more realistic clumping of leaves and branches.

The simulated scene differed from actual forest as it included a small forest patch in an open area. Therefore, care must be taken when extrapolating the results to a forest canopy. However, we believe the scaling results would not be very 
different if cyclic boundary conditions (i.e., continuous forest cover) had been used.

The SIF modeling method presented in this paper has the potential to simulate even more complex cases. For example vertical and horizontal gradients in leaf level biophysical parameters could be assigned, which are known to occur in actual canopies in response to light environment (Atherton et al., 2017). Additionally, background and branch influences on canopy SIF simulation can also be simulated based on this method.

Although some of the parameters in this study are from field data, several assumptions of the model configuration were made to interpret the canopy structure. For example the 3-D grass layer was created with parameters not based on measurements. Our method and simulations will be next validated using hyperspectral data measured from a done platform (Honkavaara et al., 2016) equipped with a Piccolo Doppio system (Mac-Arthur et al., 2014) which has been collected over spring and summer 2017. The Piccolo Doppio uses a QePro Ocean Optics Spectrometer for retrieval of canopy SIF in the oxygen B and A bands. In addition, empirical estimations of fluorescence yield, leaf biochemistry, and PSI and PSII spectra will be used instead of synthetic data in following steps.

\section{ACKNOWLEDGEMENT}

This research has been co-financed by the UCAS (UCAS [2015] 37) Joint PhD Training Program and the Academy of Finland (Grant \# 293443).

\section{REFERENCES}

Alonso L, Gomez-Chova L, Vila-Frances J, et al. Improved Fraunhofer Line Discrimination method for vegetation fluorescence quantification[J]. IEEE Geoscience and Remote Sensing Letters, 2008, 5(4): 620-624.

Amoros-Lopez J, Gomez-Chova L, Vila-Frances J, et al. Evaluation of remote sensing of vegetation fluorescence by the analysis of diurnal cycles [J]. International Journal of Remote Sensing, 2008, 29(17-18): 5423-5436.

Alonso L, Van Wittenberghe S, Amorós-López J, et al. Diurnal Cycle Relationships between Passive Fluorescence, PRI and NPQ of Vegetation in a Controlled Stress Experiment[J]. Remote Sensing, 2017, 9(8): 770.

Atherton J, Olascoaga B, Alonso L, et al. Spatial variation of leaf optical properties in a boreal forest is influenced by species and light environment [J]. Frontiers in plant science, 2017, 8 .

Brito, A. (2008). Blender 3D: Architecture, Buildings, and Scenery: Create photorealistic 3D architectural visualizations of buildings, interiors, and environmental scenery. Packt Publishing Ltd.

Damm A, Guanter L, Paul-Limoges E, et al. Far-red suninduced chlorophyll fluorescence shows ecosystem-specific relationships to gross primary production: An assessment based on observational and modeling approaches [J]. Remote Sensing of Environment, 2015, 166: 91-105.

Guillevic P, Gastellu-Etchegorry J P. Modeling BRF and radiation regime of boreal and tropical forest: II. PAR regime [J]. Remote Sensing of Environment, 1999, 68(3): 317-340.

Gastellu-Etchegorry, J. P., Lauret, N., Yin, T., Landier, L., Kallel, A., Malenovský, Z., \& Medjdoub, G. (2017). DART: Recent Advances in Remote Sensing Data Modeling With Atmosphere, Polarization, and Chlorophyll Fluorescence. IEEE Journal of Selected Topics in Applied Earth Observations and Remote Sensing.

Honkavaara, E., Hakala, T., Nevalainen, O., Viljanen, N., Rosnell, T., Khoramshahi, E., \& Tommaselli, A. (2016). Geometric and Reflectance Signature Characterization of Complex Canopies Using Hyperspectral Stereoscopic Images from Uav and Terrestrial Platforms. ISPRS-International Archives of the Photogrammetry, Remote Sensing and Spatial Information Sciences, 41, B7.

Hernández-Clemente R, North $\mathrm{P} \mathrm{R} \mathrm{J}$, Hornero $\mathrm{A}$, et al. Assessing the effects of forest health on sun-induced chlorophyll fluorescence using the FluorFLIGHT 3-D radiative transfer model to account for forest structure [J]. Remote Sensing of Environment, 2017, 193: 165-179.

Jacquemoud S, Verhoef W, Baret F, et al. PROSPECT+ SAIL models: A review of use for vegetation characterization [J]. Remote sensing of environment, 2009, 113: S56-S66.

Miller J R, Berger M, Jacquemoud S, et al. Overview of FluorMOD: A project to develop an integrated leaf-canopy fluorescence simulation model[C]//2nd International Workshop on Remote Sensing of Vegetation Fluorescence, 17-19 November, 2004, Montreal (Canada). 2004.

Malenovský Z, Martin E, Homolová L, et al. Influence of woody elements of a Norway spruce canopy on nadir reflectance simulated by the DART model at very high spatial resolution[J]. Remote Sensing of Environment, 2008, 112(1): 118.

Meroni M, Rossini M, Guanter L, et al. Remote sensing of solar-induced chlorophyll fluorescence: Review of methods and applications [J]. Remote Sensing of Environment, 2009, 113(10): 2037-2051.

Mac Arthur, A., Robinson, I., Rossini, M., Davis, N., \& MacDonald, K. (2014). A dual-field-of-view spectrometer system for reflectance and fluorescence measurements (Piccolo Doppio) and correction of etaloning. In Proceedings of the Fifth International Workshop on Remote Sensing of Vegetation Fluorescence (pp. 22-24).

Migliavacca, M., Perez-Priego, O., Rossini, M., El-Madany, T. S., Moreno, G., van der Tol, C., \& Carrara, A. (2017). Plant functional traits and canopy structure control the relationship between photosynthetic $\mathrm{CO} 2$ uptake and far-red sun-induced fluorescence in a Mediterranean grassland under different nutrient availability. New Phytologist, 214(3), 1078-1091. 
North P R J. Three-dimensional forest light interaction model using a Monte Carlo method [J]. IEEE transactions on Geoscience and Remote Sensing, 1996, 34(4): 946-956.

Porcar-Castell, A., Tyystjärvi, E., Atherton, J., van der Tol, C., Flexas, J., Pfündel, E. E., ... \& Berry, J. A. (2014). Linking chlorophyll a fluorescence to photosynthesis for remote sensing applications: mechanisms and challenges. Journal of experimental botany, eru191.

Van der Tol, Verhoef W, Timmermans J, et al. An integrated model of soil-canopy spectral radiances, photosynthesis, fluorescence, temperature and energy balance $[\mathrm{J}]$. Biogeosciences, 2009, 6(12): 3109-3129.

Verhoef W. Extension of SAIL to model solar-induced canopy fluorescence spectra $[\mathrm{M}] / /$ Processdings of the 2nd international workshop on remote sensing of vegetation fluorescence, 17-19 Nov. 2004. ESA, 2004, Montreal, Canada/Paris (ESA WPP; 242, 18p.).

Verhoef W. Modeling vegetation fluorescence observations[C] //Proceedings of the EARSel 7th SIG-Imaging Spectroscopy Workshop, Edinburgh, UK. 2011: 11-13.

Verrelst J, Rivera J P, van der Tol C, et al. Global sensitivity analysis of the SCOPE model: What drives simulated canopyleaving sun-induced fluorescence? [J]. Remote Sensing of Environment, 2015, 166: 8-21.

Van Wittenberghe S, Alonso L, Verrelst J, et al. Bidirectional sun-induced chlorophyll fluorescence emission is influenced by leaf structure and light scattering properties-A bottom-up approach[J]. Remote Sensing of Environment, 2015, 158: 169179.

Widlowski J L, Mio C, Disney M, et al. The fourth phase of the radiative transfer model intercomparison (RAMI) exercise: Actual canopy scenarios and conformity testing [J]. Remote Sensing of Environment, 2015, 169: 418-437.

Yang X, Tang J, Mustard J F, et al. Solar-induced chlorophyll fluorescence that correlates with canopy photosynthesis on diurnal and seasonal scales in a temperate deciduous forest [J]. Geophysical Research Letters, 2015, 42(8): 2977-2987.

Zhao F, Dai X, Verhoef W, et al. FluorWPS: A Monte Carlo ray-tracing model to compute sun-induced chlorophyll fluorescence of three-dimensional canopy [J]. Remote Sensing of Environment, 2016, 187: 385-399. 